07

\title{
Исследование влияния случайных флуктуаций концентрации легирующей примеси на ток в полупроводниковых сверхрешетках
}

\author{
(C) А.О. Сельский ${ }^{1,2}$, А.А. Короновский ${ }^{1}$, О.И. Москаленко ${ }^{1}$, \\ A.E. Храмов ${ }^{2}$
}

${ }^{1}$ Саратовский национальный исследовательский государственный университет им. Н.Г. Чернышевского

${ }^{2}$ Саратовский государственный технический университет

им. Гагарина Ю.А.

E-mail: feanorberserk@gmail.com

Поступило в Редакцию 1 июня 2017 г.

Исследуется, как случайные флуктуации концентрации легирующей примеси влияют на вольт-амперные характеристики тока, протекающего через полупроводниковую сверхрешетку. Показано, что в зависимости от амплитуды флуктуаций параметров наноструктуры характеристики тока, протекающего через сверхрешетку, заметно изменяются. По малой выборке удалось найти закон распределения плотности вероятности значений интеграла модуля разности токов при различных значениях амплитуды флуктуаций концентрации легирующей примеси.

DOI: 10.21883/PJTF.2017.20.45144.16896

Объектом исследования в настоящей работе является гетероструктура, состоящая из чередующихся слоев (ширина слоя, как правило, не превышает нескольких десятков нанометров) различных полупроводниковых материалов с близкими периодами кристаллической ре- 
шетки, - полупроводниковая сверхрешетка $[1,2]$. Сверхрешетки служат удобной моделью для изучения различных квантово-механических эффектов $[3,4]$. Не меньший интерес вызывает коллективная динамика электронов при приложении к сверхрешетке постоянного напряжения, поскольку в этом случае в структуре могут образовываться пространственно-временны́е электронные структуры, называемые доменами (по аналогии с доменами в диоде Ганна $[5,6]$ ). Прохождение доменов через полупроводниковую сверхрешетку приводит к возникновению колебаний тока, протекающего через структуру, частота которых составляет, как правило, несколько десятков гигагерц $[7,8]$. Следует отметить, что в настоящее время все больше внимания исследователи уделяют рассмотрению транспорта электронов через полупроводниковую сверхрешетку с позиций нелинейной динамики. Это позволяет выявлять и объяснять различные эффекты, наблюдающиеся в исследуемой системе [7-11].

Важным фактом является то, что при создании полупроводниковых гетероструктур возможно возникновение большого числа различных пространственных неоднородностей (например, случайных флуктуаций концентрации легирующей примеси $[12,13])$, которые оказывают влияние на характеристики сверхрешетки. Помимо случайных флуктуаций возможно целенаправленное изменение концентрации легирующей примеси для улучшения характеристик прибора $[14,15]$. В настоящей работе исследован вопрос, как уровень случайных флуктуаций концентрации легирующей примеси влияет на характеристики полупроводниковой сверхрешетки.

Типовой моделью при описании процессов транспорта заряда в полупроводниковой сверхрешетке является система уравнений, включающая в себя уравнение непрерывности, уравнение Пуассона и выражение для плотности тока с учетом дрейфовой скорости электрона $[2,9]$ :

$$
\begin{gathered}
e \frac{\partial n}{\partial t}=\frac{\partial J}{\partial x}, \\
\frac{\partial F}{\partial x}=\frac{e}{\varepsilon_{0} \varepsilon_{r}}\left(n-n_{D 0}\right), \\
J=e n v_{d}(\bar{F}),
\end{gathered}
$$

где $t$ обозначает время, а координата $x$ соответствует направлению движения электронов в полупроводниковой сверхрешетке. Величины 
$n(x, t), F(x, t)$ и $J(x, t)$ определяют концентрацию, напряженность электрического поля и плотность тока соответственно. Параметры $\varepsilon_{0}, \varepsilon_{r}=12.5$ отвечают абсолютной и относительной диэлектрическим проницаемостям; $n_{D 0}=3 \cdot 10^{22} \mathrm{~m}^{-3}-$ равновесная концентрация электронов; $v_{d}$ - дрейфовая скорость электрона, вычисленная для среднего значения напряженности электрического поля $\bar{F} ; e>0-$ заряд электрона. Зависимость дрейфовой скорости, входящей в уравнение (1), от напряженности электрического поля определяется по формуле Эсаки-Тсу [4].

Будем исходить из предположения, что в каждом слое величина концентрации легирующей примеси отличается от равновесного значения $n_{D 0}$ на случайную величину [12]. Тогда для учета случайных флуктуаций модифицируем уравнение Пуассона из (1) следующим образом:

$$
\frac{\partial F}{\partial x}=\frac{e}{\varepsilon_{0} \varepsilon_{r}}\left[n-\left(n_{D 0}+D n_{D 1}(x)\right)\right]
$$

где $n_{D 1}(x)$ - равномерное распределение случайной величины в диапазоне $[-0.5 ; 0.5]$ в соответствии с $[12], D-$ амплитуда флуктуаций концентрации легирующей примеси.

Важной характеристикой коллективной динамики электронов является вольт-амперная характеристика (BAX). Начало падающего участка на ВАХ соответствует возникновению генерации колебаний тока. Для построения BAX на этом участке значение тока усреднялось по времени. Метод построения вольт-амперных характеристик и их вид для случая без учета флуктуаций концентрации легирующей примеси $\left(n_{D 1}(x)=0\right.$ вдоль всей сверхрешетки) описан в [11] (рис. 1). Из приведенного рисунка можно видеть, что для различных профилей концентрации легирующей примеси (при одной и той же амплитуде флуктуаций $D$ ) вольт-амперные характеристики отклоняются от случая, когда флуктуаций нет.

Введем величину $\Theta$, определяющую отклонение ВАХ от случая без флуктуаций концентрации легирующей примеси и рассчитываемую следующим образом:

$$
\Theta=\frac{\int\left|I(V)-I_{0}(V)\right| d V}{\int I_{0}(V) d V}
$$

где $I(V)$ - значение тока при напряжении $V ; I_{0}(V)$ - значение тока при данном напряжении в случае отсутствия флуктуаций (амплитуда

Письма в ЖТФ, 2017, том 43, вып. 20 


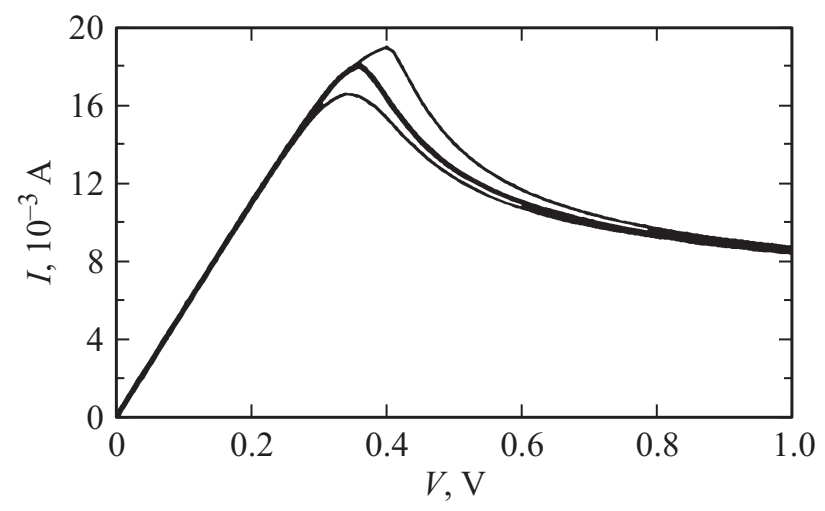

Рис. 1. Примеры вольт-амперных характеристик для нескольких профилей концентрации легирующей примеси при значении амплитуды флуктуаций $D=0.5$ (жирная линия — случай без флуктуаций).

флуктуаций $D$ стремится к нулю). Данная величина будет различной для каждого профиля со случайными флуктуациями концентрации легирующей примеси.

На практике при создании реальных приборов, в том числе и на основе полупроводниковых сверхрешеток, важно оценить отклонение характеристик при фиксированной величине амплитуды флуктуаций параметров прибора (считая, что она зависит от технологического процесса). Для получения данной оценки важными являются методы нахождения по малой выборке распределения характеристик прибора, что представляется разумным с точки зрения экономии при создании прототипов приборов. В ходе компьютерного моделирования также возникает проблема нахождения по малой выборке вида распределения характеристик, так как каждый расчет параметров прибора требует существенных затрат машинного времени. Решить данную проблему можно с помощью ранжированного распределения. Ранжированное распределение более удобно в том плане, что в этом случае не происходит редукции числа данных, как при построении соответствующей гистограммы [16,17]. Ранжированные распределения для 200 случайных профилей и разных значений амплитуды, полученных с помощью численного моделирования, показаны на рис. 2, $a$. Наименьшему зна- 

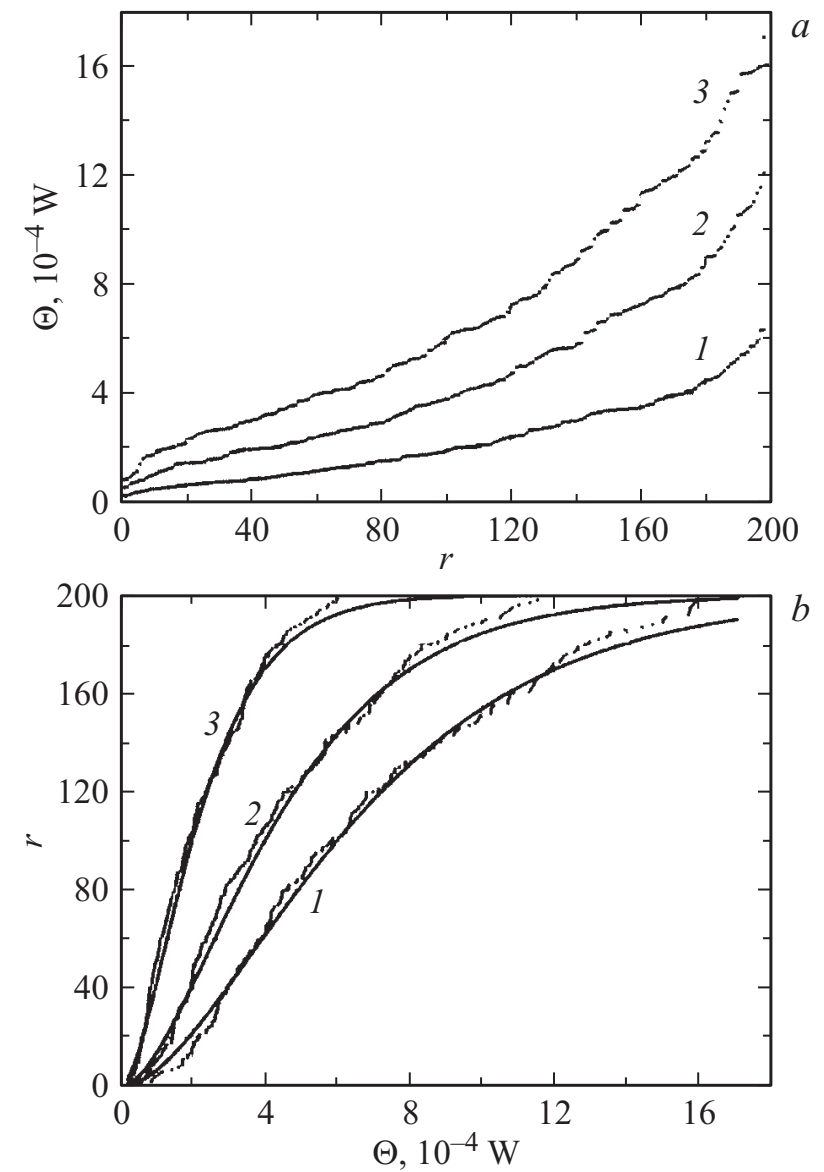

Рис. 2. $a-$ упорядоченные в порядке возрастания значения интеграла модуля разности токов $\Theta$ для различных амплитуд флуктуаций $D ; b-$ ранжированные распределения значений интеграла модуля разности токов $\Theta$ для различных амплитуд флуктуаций $D . D=0.25(1), 0.5$ (2), 0.75 (3).

чению величины $\Theta$ присваивается ранг 1, следующей по значению величине - ранг 2 , и так далее до наибольшего значения ранга у максимального значения величины $\Theta$. Для большого числа событий ранг

Письма в ЖТФ, 2017, том 43, вып. 20 

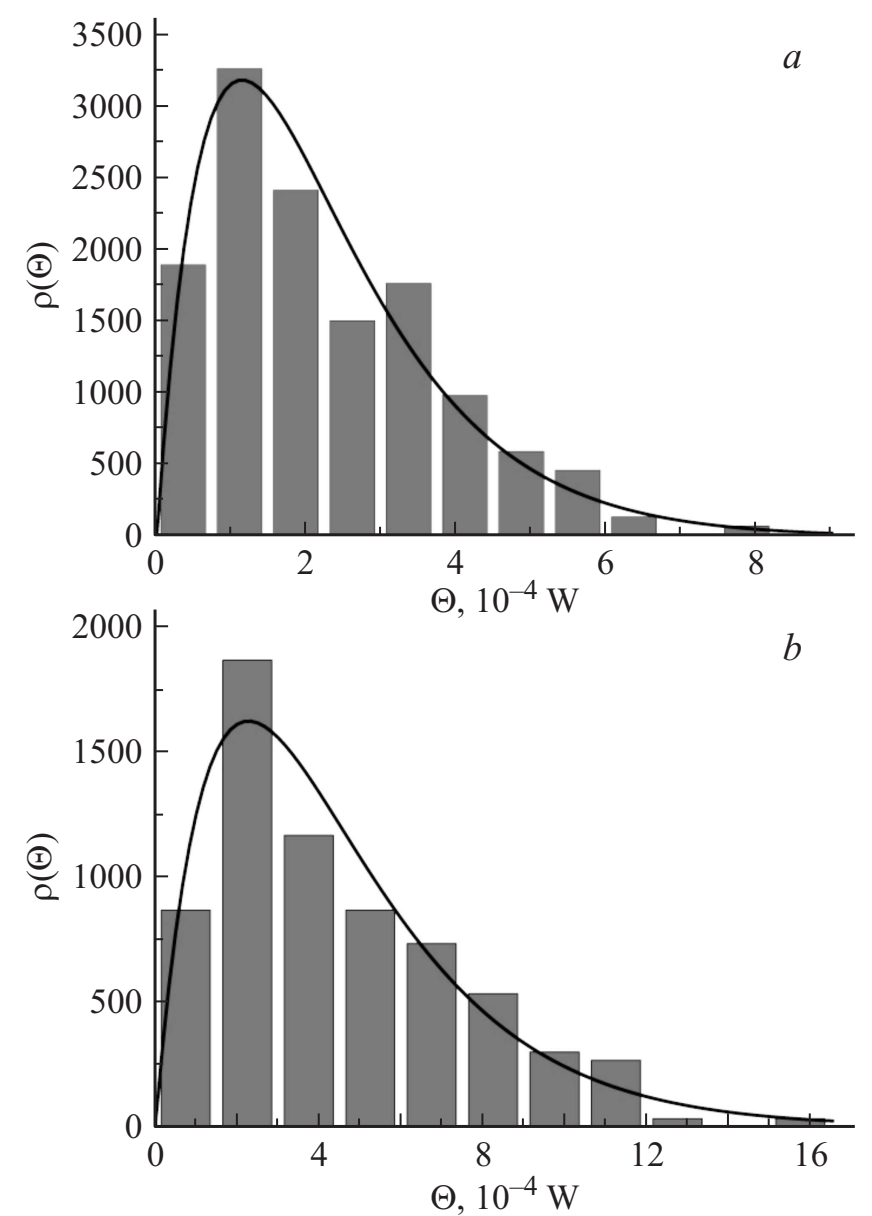

Рис. 3. Распределения плотности вероятности значений интеграла модуля разности токов $\Theta$ для различных амплитуд флуктуаций $D: a-0.25, b-0.5$, $c-0.75$.

можно считать непрерывной величиной. Тогда, опираясь на описанную в работе [18] идеологию, можно найти, как ранжированное распределение величины $\Theta$ связано с распределением плотности вероятности 


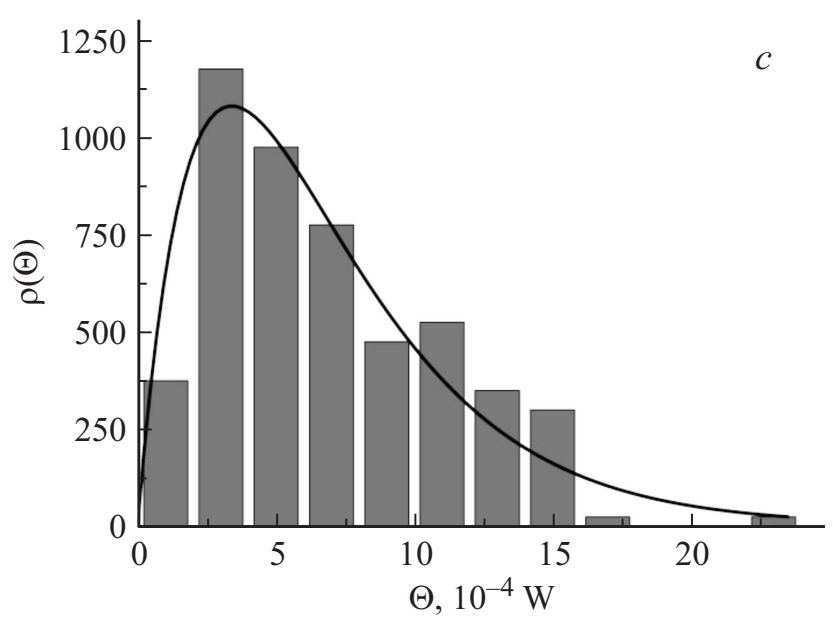

Рис. 3 (продолжсение).

величины $\Theta$, а именно

$$
\rho(\Theta)=d r / d \Theta,
$$

где $r$ - ранг. На рис. 2, $b$ ранжированные распределения показаны как функции величины $\Theta$, что соответствует выражению (4) и упрощает дальнейший анализ. В данном виде по зависимостям ранга от величины $\Theta$ легко можно найти аппроксимацию, которая имеет следующий вид:

$$
r=N(1-\exp (-b \Theta / D)(1+b \Theta / D)) .
$$

Здесь $N=200$ - общее число случайных профилей, $b=2200-$ постоянный параметр. На рис. $2, b$ данные аппроксимации для различных значений амплитуды флуктуаций $D$ показаны линиями, при этом наблюдается хорошее согласие между аппроксимацией и ранжированными зависимостями. Теперь, используя (5), можно найти закон распределения плотности вероятности величины $\Theta$ с помощью соотношения (4). Легко видеть, что он будет иметь вид

$$
\rho(\Theta)=\Theta \exp (-b \Theta / D)(b / D)^{2} .
$$

На рис. 3 полученные численно распределения плотности вероятности величины $\Theta$ показаны в виде гистограмм при различных

Письма в ЖТФ, 2017, том 43, вып. 20 
значениях амплитуды флуктуаций $D$. Линиями на рисунке представлены кривые, соответствующие формуле (6). Можно видеть, что в целом для столь малой выборки численно найденные распределения плотности вероятности величины $\Theta$ неплохо согласуются с полученными ранее законами для разных амплитуд флуктуаций (6).

Таким образом, в работе показано, как случайные флуктуации концентрации легирующей примеси влияют на вольт-амперные характеристики в полупроводниковой сверхрешетке. Для учета изменений была введена интегральная величина $\Theta$ и исследовано, как она зависит от амплитуды флуктуаций концентрации легирующих примесей. По относительно небольшой выборке профилей концентрации легирующей примеси (200 профилей при фиксированном уровне случайных флуктуаций) с помощью ранжирования данных был получен вид распределения плотности вероятности величины $\Theta$ для произвольного значения амплитуды флуктуаций. Предложенный метод можно применять к небольшим выборкам прототипов реальных приборов, в том числе на основе полупроводниковых сверхрешеток, для нахождения распределения отклонений характеристик при случайных флуктуациях параметров, возникающих при создании приборов.

Работа выполнена при поддержке Российского фонда фундаментальных исследований (проект № 16-32-60059).

\section{Список литературы}

[1] Шик А.Я. // ФТП. 1974. Т. 8. С. 1841-1864.

[2] Wacker A. // Phys. Rep. 2002. V. 357. P. 1-111.

[3] Келдыш Л.В. // ФТТ. 1962. Т. 4. С. 2265-2267.

[4] Esaki L., Tsu R. // IBM J. Res. Dev. 1970. V. 14. P. 61-65.

[5] Scheuerer R., Schomburg E., Renk K.F. et al. // Appl. Phys. Lett. 2002. V. 81. P. $1515-1517$.

[6] Eisele H., Khanna S.P., Lineld E.H. // Appl. Phys. Lett. 2010. V. 96. P. 072101.

[7] Bonilla L.L., Grahn H.T. // Rep. Prog. Phys. 2005. V. 68. P. 577-683.

[8] Fromhold T. M., Patanè A., Bujkiewicz S. et al. // Nature. 2004. V. 428. P. 726730.

[9] Selskii A.O., Koronovskii A.A., Hramov A.E. et al. // Phys. Rev. B. 2011. V. 84. P. 235311.

[10] Bonilla L.L., Teitsworth S.W. Nonlinear wave methods for charge transport. Weinheim: WILEY-VCH Verlag GmbH \& Co. KGaA, 2010. 287 p.

Письма в ЖТФ, 2017, том 43, вып. 20 
[11] Hramov A.E., Makarov V.V., Koronovskii A.A. et al. // Phys. Rev. Lett. 2014. V. 112. P. 116603.

[12] Wacker A., Schwarz G., Prengel F. et al. // Phys. Rev. B. 1995. V. 52. P. 13788.

[13] Patra M., Schwarz G., Scholl E. // Phys. Rev. B. 1998. V. 57. P. 1824.

[14] Баланов А.Г., Короновский А.А., Москаленко О.И. и др. // Изв. РАН. Сер. физ. 2017. Т. 81. № 1. С. 50-54.

[15] Баланов А.Г., Короновский А.А., Москаленко О.И. и др. // Уч. зап. физ. фак-та МГУ. 2016. Т. 6. С. 166907.

[16] Короновский А.А., Трубецков Д.И. // Нелинейная динамика в действии. Саратов: Изд-во ГосУНЦ „Колледж“, 2002. 324 р.

[17] Zipf G.K. // Human behavior and the principle of least effort. Addison-Wesley, 1949. $585 \mathrm{p}$.

[18] Newman M.E.J. // Proc. Roy. Soc. London. B. 1996. V. 263. P. 1605-1610.

Письма в ЖТФ, 2017, том 43, вып. 20 